\title{
Optimization of single-walled carbon nanotube solubility by noncovalent PEGylation using experimental design methods
}

This article was published in the following Dove Press journal:

International Journal of Nanomedicine

7 April 201 I

Number of times this article has been viewed

\author{
Naghmeh Hadidi' \\ Farzad Kobarfard ${ }^{2}$ \\ Nastaran Nafissi-Varcheh ${ }^{3}$ \\ Reza Aboofazeli' \\ 'Department of Pharmaceutics, \\ ${ }^{2}$ Department of Pharmaceutical \\ Chemistry, ${ }^{3}$ Department of \\ Pharmaceutical Biotechnology, School \\ of Pharmacy, Shaheed Beheshti \\ University of Medical Sciences, Tehran, \\ Iran
}

\begin{abstract}
In this study, noncovalent functionalization of single-walled carbon nanotubes (SWCNTs) with phospholipid-polyethylene glycols (Pl-PEGs) was performed to improve the solubility of SWCNTs in aqueous solution. Two kinds of PEG derivatives, ie, Pl-PEG 2000 and P1-PEG 5000, were used for the PEGylation process. An experimental design technique (D-optimal design and second-order polynomial equations) was applied to investigate the effect of variables on PEGylation and the solubility of SWCNTs. The type of PEG derivative was selected as a qualitative parameter, and the PEG/SWCNT weight ratio and sonication time were applied as quantitative variables for the experimental design. Optimization was performed for two responses, aqueous solubility and loading efficiency. The grafting of PEG to the carbon nanostructure was determined by thermogravimetric analysis, Raman spectroscopy, and scanning electron microscopy. Aqueous solubility and loading efficiency were determined by ultraviolet-visible spectrophotometry and measurement of free amine groups, respectively. Results showed that Pl-PEGs were grafted onto SWCNTs. Aqueous solubility of $0.84 \mathrm{mg} / \mathrm{mL}$ and loading efficiency of nearly $98 \%$ were achieved for the prepared Pl-PEG 5000-SWCNT conjugates. Evaluation of functionalized SWCNTs showed that our noncovalent functionalization protocol could considerably increase aqueous solubility, which is an essential criterion in the design of a carbon nanotube-based drug delivery system and its biodistribution.
\end{abstract}

Keywords: phospholipid-PEG, D-optimal design, loading efficiency, Raman spectroscopy, scanning electron microscopy, theromogravimetric analysis, carbon nanotubes

\section{Introduction}

Particle engineering and design of nanoscale drug delivery systems with uniform particle size, desired surface characteristics and geometrical forms have considerably advanced over the past few years. The great advantage of nanotechnology is its potential capability of creating unique structures with unique characteristics, leading to the improvement of membrane penetration, an increase in solubility of poorly soluble drugs, stability of biomolecules and their bioavailabilities, and consequently improvement of drug delivery efficiency.

One of the most recent strategies proposed to incorporate nanotechnology principles is through the application of carbon nanotubes (CNTs). CNTs (hollow cylinders composed of rolled sheets of graphene built from a hexagonal arrangement of $\mathrm{sp}^{2}$-hybridized carbon atoms in nanoscale dimensions) ${ }^{1}$ were first introduced by Iijima ${ }^{2}$ in 1991 and have displayed unique structures and remarkable mechanical, thermal, magnetic, optical, electrical, surface, and chemical properties, such that the combination of these characteristics provides extensive biomedical and pharmaceutical
Correspondence: Reza Aboofazel Department of Pharmaceutics, School of Pharmacy, Shaheed Behesht University of Medical Sciences,

Vali Asr Avenue, Niayesh Junction,

Tehran 199195338I, Iran

Tel/Fax +98 2I $8820007 \mid$

Email raboofazeli@sbmu.ac.ir 
applications. ${ }^{3-6}$ However, CNTs are inert and intrinsically poorly soluble in nearly all organic and inorganic solvents and physiologic fluids. ${ }^{7}$ To be biocompatible, the aqueous solubility of CNTs is a prerequisite. Therefore, in order to be used in drug delivery, they should meet this requirement. ${ }^{8}$ Among the various approaches used to obtain an appropriate dispersion, functionalization of CNT sidewalls, either covalently or noncovalently, has attracted much attention. ${ }^{7,9-11}$ It has been shown that following this process, an increase in CNT solubility can be achieved. The possibility of functionalization enables the introduction of several pharmaceutically active ingredients simultaneously, creating CNT conjugates endowed with some kind of pharmacological activity.

The aim of the main project was to use CNT-based nanostructures as efficient carriers for drug loading and delivery. As the first step, to overcome the problems associated with the solubility of single-walled carbon nanotubes (SWCNTs), the present study was planned to functionalize SWCNTs noncovalently with two commercially available phospholipidpolyethylene glycols (Pl-PEG 2000 and Pl-PEG 5000) and evaluate the effect of PEGylation on their solubility. We hypothesized that through noncovalent functionalization with Pl-PEGs, the conjugate would be readily cleaved to generate the active agent. Experimental design techniques were then applied to investigate the effect of variables on PEGylation and solubility. Optimization was finally performed for two responses, aqueous solubility and PEG loading. The attachment of PEGs to the carbon nanostructures was evaluated by thermogravimetric analysis (TGA), Raman spectroscopy, and scanning electron microscopy (SEM), and water solubility and loading efficiency were determined by ultraviolet-visible (UV-Vis) spectrophotometry and the measurement of free amine groups, respectively.

\section{Materials and methods Materials}

PEG-SWCNT (P7-SWCNT) with $\mathrm{D}_{50}$ of $2.48 \mathrm{~nm}$ and PEG content of $29 \%$ and pure SWCNT (P2-SWCNT) with $\mathrm{D}_{50}$ of $4.03 \mathrm{~nm}$, carbonaceous purity of $\geq 90 \%$, metal content of $4 \%-7 \%$, and prepared by chemical vaporization deposition technique were purchased as the standards from Carbon Solu-

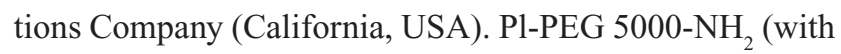
a molecular weight of 5679) and Pl-PEG 2000- $\mathrm{NH}_{2}$ (with a molecular weight of 2679) were prepared by NOF-Sunbright Company (New York, USA) and Avanti Lipid Company (Alabama, USA), respectively, and used for the functionalization process. 2,4,6-trinitrobenzene sulfonic acid (TNBSA) was obtained from Thermo Fisher Scientific Company (Illinois, USA) and used for the determination of free amines. All other reagents were analytical grade.

\section{Methods}

\section{Noncovalent functionalization with PI-PEGs}

Appropriate amounts of Pl-PEG 2000- $\mathrm{NH}_{2}$ and Pl-PEG 5000- $\mathrm{NH}_{2}$ (based on the PEG/SWCNT weight ratio, shown in Table 1) were weighed and transferred into glass vials containing $2 \mathrm{~mL}$ deionized water and completely dissolved by gentle shaking. SWCNTs (1 mg) were also weighed and transferred into glass vials containing $2 \mathrm{~mL}$ deionized water and sonicated for 5 minutes at room temperature. Dissolved Pl-PEGs were added to partially dispersed SWCNTs, and sonication was then continued for defined periods at room temperature. The obtained suspension was centrifuged (Optima L-90 K, Beckman Coulter, California, USA) at $18,000 \mathrm{rpm}$ for 6 hours at room temperature to separate functionalized, soluble SWCNTs ( $f$-SWCNTs) as the supernatant. $0.5 \mathrm{~mL}$ of the supernatant was transferred into a $2 \mathrm{~mL}$ Vivaspin centrifugal filter device with a molecular weight cut-off of $100 \mathrm{kDa}$. $1.5 \mathrm{~mL}$ deionized water was added and the mixture was centrifuged at $5000 \mathrm{rpm}$ for 15 minutes at room temperature. The precipitate was then washed with deionized water five to six times in order to completely remove the excess of Pl-PEGs.

\section{Experimental design methods}

The functionalization process was designed based on one qualitative variable (type of Pl-PEG) and two quantitative variables (P1-PEG/SWCNT weight ratio and sonication time), using a D-optimal design method in two blocks. The quantitative variables and their constraints were chosen based on previous work on CNT PEGylation (Table 2). ${ }^{12}$ Design-Expert ${ }^{\circledR}$ software (Version 7.0.0, Stat-Ease, Inc. Minneapolis, USA) was used to analyze the solubility of SWCNTs (response 1), PEGylation efficiency (response 2), and all model variables. A suitable polynomial model was selected based on significant terms $(P<0.05)$, coefficient of variation $(\mathrm{CV})$, multiple correlation coefficient $\left(R^{2}\right)$, and adjusted multiple correlation coefficient (adjusted $R^{2}$ ). Optimization of functionalization was performed by maximization of both responses, and a contour plot was finally constructed to indicate the domain for the samples with the desirability index of 1 and desirable values for both responses, simultaneously. To verify the optimization, four optimized samples were prepared experimentally, and both responses were evaluated. 
Table I Experimental runs designed by D-optimal model, using qualitative (phospholipid-polyethylene glycol [PI-PEG] type) and quantitative variables (single-walled carbon nanotube [SWCNT] aqueous concentration and loading efficiency) on the basis of two responses

\begin{tabular}{|c|c|c|c|c|c|}
\hline \multirow[t]{2}{*}{ Run } & \multicolumn{3}{|c|}{ D-optimal design variables } & \multicolumn{2}{|l|}{ Measured responses } \\
\hline & $\begin{array}{l}\text { PI-PEG/SWCNT } \\
\text { weight ratio }(\mathrm{A})\end{array}$ & $\begin{array}{l}\text { Sonication } \\
\text { time (minutes) (B) }\end{array}$ & $\begin{array}{l}\text { PI-PEG } \\
\text { type (C) }\end{array}$ & $\begin{array}{l}\text { SWCNT aqueous } \\
\text { concentration }(\mathrm{mg} / \mathrm{mL})\end{array}$ & $\begin{array}{l}\text { Loading } \\
\text { efficiency (\%) }\end{array}$ \\
\hline 7 & 10.00 & 60.00 & 2000 & 0.23 & 95.31 \\
\hline 3 & 5.00 & 60.00 & 5000 & 0.21 & 91.57 \\
\hline 5 & 5.00 & 120.00 & 2000 & 0.04 & 52.62 \\
\hline 4 & 10.00 & 92.47 & 5000 & 0.31 & 60.93 \\
\hline I & 7.89 & 120.00 & 5000 & 0.58 & 5.31 \\
\hline 13 & 5.47 & 95.79 & 5000 & 0.52 & 92.33 \\
\hline 15 & 9.09 & 60.00 & 5000 & 0.77 & 26.53 \\
\hline 18 & 5.00 & 60.00 & 2000 & 0.24 & 16.20 \\
\hline 19 & 5.00 & 114.63 & 5000 & 0.12 & 30.01 \\
\hline II & 10.00 & 120.00 & 2000 & 0.32 & 50.77 \\
\hline 9 & 5.00 & 60.00 & 2000 & 0.10 & 78.99 \\
\hline 2 & 8.75 & 109.25 & 2000 & 0.64 & 47.92 \\
\hline 17 & 8.13 & 97.41 & 5000 & $0.7 \mathrm{I}$ & 80.90 \\
\hline 16 & 6.88 & 117.19 & 2000 & 0.36 & 97.83 \\
\hline 6 & 7.89 & 120.00 & 5000 & 0.61 & 92.20 \\
\hline 8 & 10.00 & 60.00 & 2000 & 0.14 & 74.78 \\
\hline 14 & 9.09 & 60.00 & 5000 & 0.90 & 90.16 \\
\hline 10 & 5.00 & 60.00 & 2000 & 0.13 & 70.16 \\
\hline 12 & 5.00 & 114.63 & 5000 & 0.14 & 55.60 \\
\hline 20 & 9.09 & 60.00 & 5000 & 0.84 & 95.00 \\
\hline
\end{tabular}

Note: $A, B$, and $C$ are notations used for the experimental design studies.

Abbreviation: PI-PEG, phospholipid-polyethylene glycol.

\section{Evaluation of aqueous solubility of PI-PEG-SWCNTs}

Construction of calibration curve and UV-Vis spectrophotometry

In order to use P7-SWCNT as the standard for the construction of the calibration curve, the weight ratios for PEG 2000-SWCNTs, PEG 5000-SWCNTs, and P7-SWCNTs were calculated using TGA experiments and found to be $12 \%, 47.72 \%$, and $29 \%$, respectively. Analysis of the results showed that $1.4 \mathrm{~g}$ of P7-SWCNT contains $1 \mathrm{~g}$ of SWCNT, which is equivalent to $2.62 \mathrm{~g}$ and $1.14 \mathrm{~g}$ of Pl-PEG 5000 and Pl-PEG 2000, respectively. ${ }^{13}$ UV absorbance of the equivalent amounts of each compound in water was measured (UV-Vis spectrophotometer, CECIL CE 2021, 2000 series, Cambridge, UK) and showed equal values, indicating that the absorbance is due only to the SWCNT structure (and not to the functionalizing compounds). Various P7-SWCNT aqueous concentrations (over the range of $0.01-0.14 \mathrm{mg} / \mathrm{mL}$ ) were then prepared for the construction of a standard calibration curve. By linear regression analysis of the corresponding plot, a linear curve with the following equation was obtained and used for the calculation of the aqueous concentration of $f$-SWCNTs at $256.8 \mathrm{~nm}$ (Figures 1 and 2). This experiment was performed in triplicate.

$$
\begin{aligned}
\text { Absorbance } & =13.07 \times(\text { Conc. } \mathrm{mg} / \mathrm{mL}) \\
& -0.0452\left(R^{2}=0.9985\right)
\end{aligned}
$$

\section{Evaluation of loading efficiency}

Measurement of free amines with TNBSA

TNBSA is a rapid and sensitive assay reagent for the determination of free amino groups. ${ }^{14}$ Primary amines upon reaction

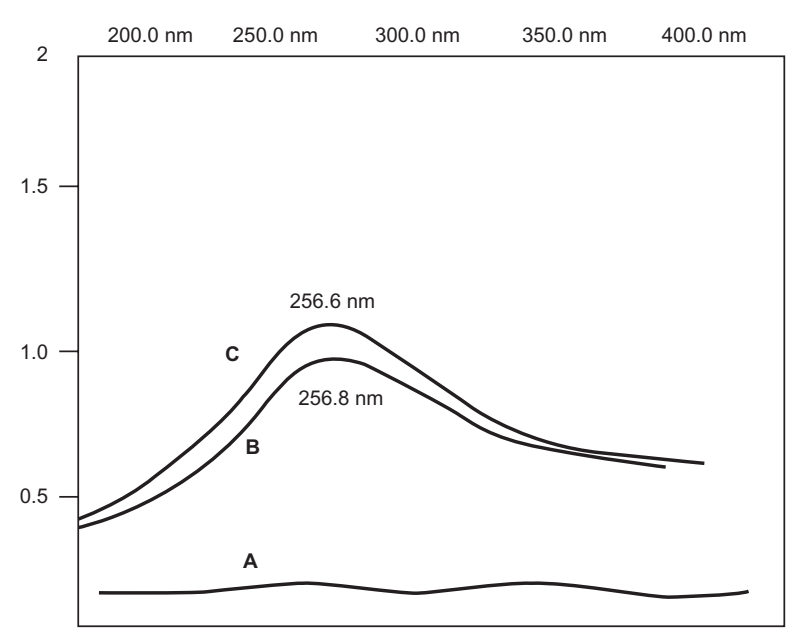

Figure I Ultraviolet-visible spectra of A) pure single-walled carbon nanotubes (SWCNTs), B) phospholipid-polyethylene glycol 5000-SWCNTs, and C) P7-SWCNTs. 


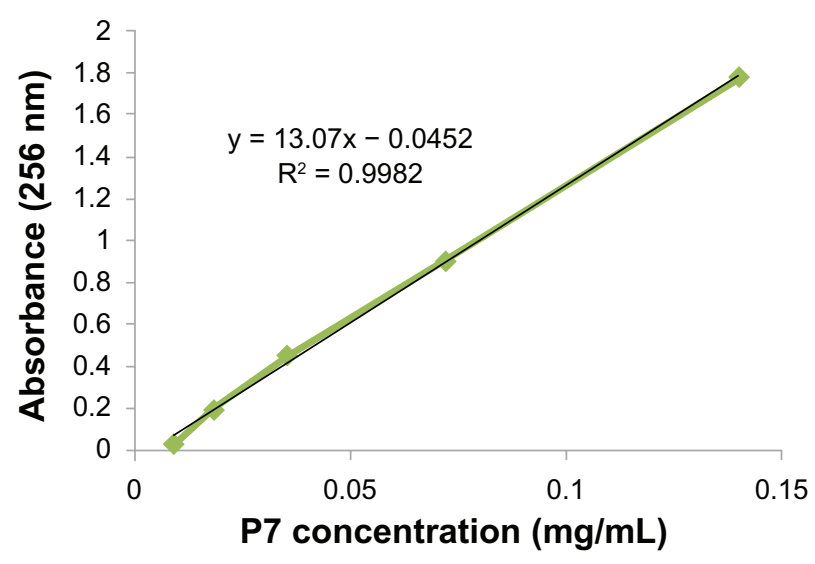

Figure 2 Ultraviolet-visible calibration curve constructed with various concentrations of P7-single-walled carbon nanotubes (SWCNTs) and used for the determination of the aqueous solubility of phospholipid-polyethylene glycol-functionalized SWCNTs.

with TNBSA form highly chromogenic derivatives that can be measured quantitatively at $335 \mathrm{~nm}$. To $0.5 \mathrm{~mL}$ aliquot of a sample ( $f$-SWCNTs), $1 \mathrm{~mL}$ of a reaction buffer $(0.1 \mathrm{M}$ sodium bicarbonate, $\mathrm{pH}=8.5)$ and $0.5 \mathrm{~mL}$ of $0.1 \%(\mathrm{w} / \mathrm{v})$ TNBSA solution were added and mixed thoroughly. After incubation at $37^{\circ} \mathrm{C}$ for 2 hours, $1 \mathrm{~mL}$ of $10 \%$ sodium dodecyl sulfate and $0.5 \mathrm{~mL}$ of $1 \mathrm{~N}$ hydrochloric acid were added, and UV absorbance was measured at $344 \mathrm{~nm}$. The free amine content was quantitatively determined using a standard calibration curve generated with known concentrations (over the range of $0.2-2.5 \mathrm{mg} / \mathrm{mL}$ ) of both amine-containing compounds. By the linear regression analysis of the corresponding plots,
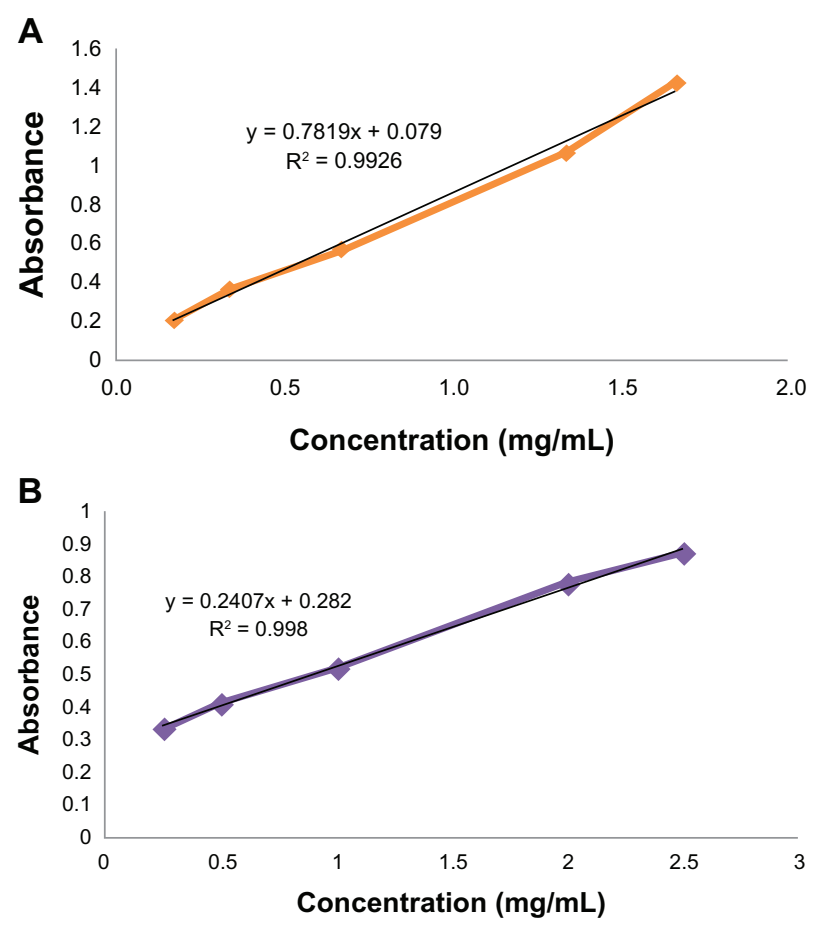

Figure 3 Ultraviolet calibration curve generated with known concentrations of both amine-containing compounds and used for the measurement of free amine content. two linear curves with the following equations were obtained and used for related calculations (Figure 3). This experiment was performed in triplicate.

For Pl-PEG 2000- $\mathrm{NH}_{2}$ Absorbance $=0.7819$
$\quad \times($ Conc $\mathrm{mg} / \mathrm{mL})+0.079\left(R^{2}=0.9926\right)$

For Pl-PEG 5000- $\mathrm{NH}_{2}$ Absorbance $=0.2407$ $\times($ Conc. $\mathrm{mg} / \mathrm{mL})+0.282\left(R^{2}=0.998\right)$

\section{Thermogravimetric analysis}

TGA was performed by TGA-50 (Shimadzu, Tokyo, Japan) for both pure SWCNTs (nonfunctionalized) and Pl-PEG-SWCNTs ( $f$-SWCNTs) up to $600^{\circ} \mathrm{C}$. PEG loading was estimated on the basis of gradual weight loss of $f$-SWCNT samples between $200^{\circ} \mathrm{C}$ and $500^{\circ} \mathrm{C}$ in comparison with nonfunctionalized samples.

\section{Raman spectroscopy}

Raman spectroscopy with a $532 \mathrm{~nm}$ laser and diffusive reflectance method (Nd:YL laser, Almega Thermo Nicolet, Florida, USA) was applied to characterize the structure of SWCNTs before and after functionalization with Pl-PEGs.

\section{Scanning electron microscopy studies}

Morphological characteristics of SWCNTs before and after functionalization with P1-PEGs were examined by SEM. SWCNTs were mounted onto aluminum stubs, vacuum coated with a gold film using a sputter coater (SCDOOS, Bal-TEC Co., Switzerland), and directly analyzed by scanning electron microscope (XL30, Philips Company, the Netherlands).

\section{Results}

D-optimal design was used for the functionalization process of SWCNTs. Table 2 summarizes the variables, types, and constraints employed. The loading efficiency and aqueous solubility of SWCNTs were assigned as dependent variables. The best-fitting mathematical model was selected based on the comparison of several statistical parameters (ie, CV, $R^{2}$, and adjusted $R^{2}$ ). Analysis of variance of both responses showed that a quadratic model was the most appropriate one $(P<0.05)$ and proved that $\mathrm{A}, \mathrm{B}, \mathrm{C}$, and $\mathrm{A}^{2}$ were significant model terms for the response 1 , and $\mathrm{A}, \mathrm{A}^{2}, \mathrm{C}$, and $\mathrm{AB}$ (not $\mathrm{B}$ and $\mathrm{B}^{2}$ ) significantly affected the response 2 . The correlation between dependent and independent variables for both responses investigated could be defined in equations 4 and 5 .

$$
\begin{aligned}
& \mathrm{Y}_{1}(\mathrm{SWCNT} \text { aqueous solubility })=0.68+0.10 \mathrm{~A} \\
& \quad-0.076 \mathrm{~B}+0.071 \mathrm{C}-0.45 \mathrm{~A}^{2} \\
& \mathrm{CV}=7.95 \%, R^{2}=0.8714, \text { adjusted } R^{2}=0.8346
\end{aligned}
$$


Table 2 Independent variables, variable types, and their constraints used for D-optimal design and optimization of functionalization process of single-walled carbon nanotubes (SWCNTs)

\begin{tabular}{lllll}
\hline Factors $^{\mathbf{a}}$ & Factor type & Lower limit & Upper limit & Goal \\
\hline PI-PEG/SWCNT weight ratio (A) & Nominal, independent & 5 & 10 & In range \\
Sonication time (minute) (B) & Nominal, independent & 60 & 120 & In range \\
PI-PEG type (C) & Categorical, independent & PI-PEG 2000-NH & PI-PEG 5000-NH & In range \\
SWCNT aqueous concentration $(\mathrm{mg} / \mathrm{mL})$ & Dependent & 0.68 & 0.85 & Maximize \\
Loading efficiency & Dependent & 75 & 95 & Maximize \\
\hline
\end{tabular}

Note: ${ }^{\mathrm{A}} \mathrm{A}, \mathrm{B}$, and $\mathrm{C}$ are notations used for the experimental design studies.

Abbreviation: PI-PEG, phospholipid-polyethylene glycol.

$$
\begin{gathered}
\mathrm{Y}_{2} \text { (loading efficiency) }=87.91+10.54 \mathrm{~A}+1.38 \mathrm{~B} \\
+9.85 \mathrm{C}+15.84 \mathrm{AB}-37.22 \mathrm{~A}^{2}+2.07 \mathrm{~B}^{2} \\
\mathrm{CV}=5.73 \%, R^{2}=0.8862, \text { adjusted } R^{2}=0.8536
\end{gathered}
$$

From equation 4 (considering the $\mathrm{Y}_{1}$ response), it was concluded that the model terms $\mathrm{A}$ and $\mathrm{C}$ have positive effects on SWCNT aqueous solubility, whereas the terms $\mathrm{A}^{2}$ and $\mathrm{B}$ affect this response negatively. From $\mathrm{Y}_{2}$, it was concluded that the model terms $\mathrm{A}, \mathrm{C}$, and $\mathrm{AB}$ have positive effects on loading efficiency, whereas $\mathrm{A}^{2}$ affects this response negatively. Experimental runs proposed by D-optimal design are summarized in Table 1, as well as the results for the quantitative measurement of aqueous concentration of SWCNTs and loading efficiency determined by UV spectrophotometry and free-amine/TNBSA reaction, respectively.

Response surface contours for aqueous concentration of SWCNTs and PEG loading are shown in Figures 4 and 5. These contour plots suggest that aqueous concentration of $0.58-0.69 \mathrm{mg} / \mathrm{mL}$ could be achieved at Pl-PEG 2000/SWCNT weight ratios of 7.54-7.96 and sonication times of 60-105 minutes (Figure 4A), whereas the PEG loading of

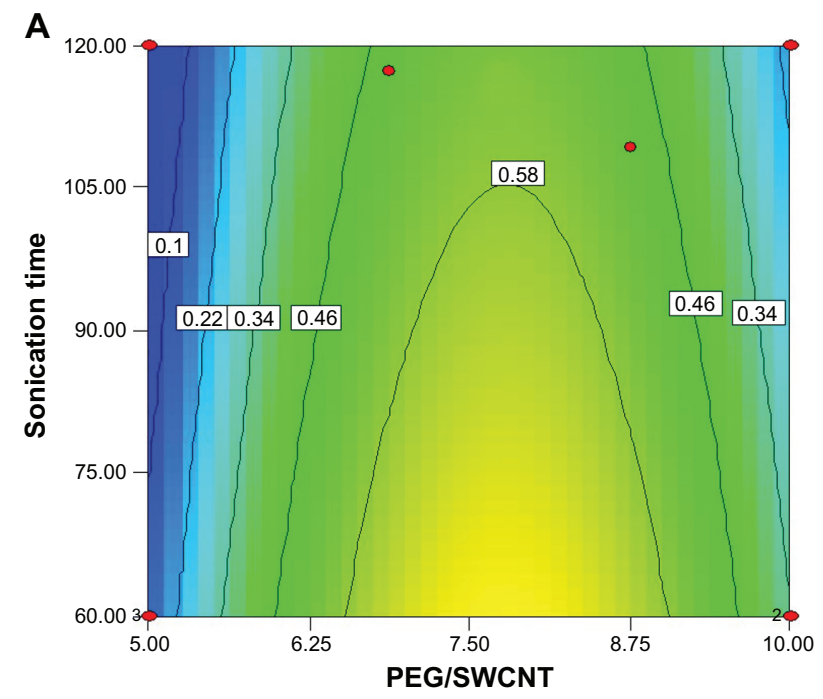

$75 \%-85 \%$ could be obtained in the weight ratios of $6.87-9.42$ and sonication times of 60-117 minutes (Figure 4B). However, for Pl-PEG 5000, water solubility of $0.58-0.83 \mathrm{mg} / \mathrm{mL}$ was achieved in the weight ratio of 7.68-9.24 and sonication times of 60-102 minutes (Figure 5A), and the PEG loading of $94 \%-98 \%$ was obtained in the ratio of $6.6-8.91$ and sonication times of 60-110 minutes (Figure 5B).

As mentioned previously, PEG-grafted SWCNT precipitates were washed five to six times in order to completely remove the excess of free Pl-PEGs. It was observed that the unbound Pl-PEGs can be removed nearly completely if the samples are washed five times.

Figure 6 shows the Raman spectra of pure SWCNTs, Pl-PEG 2000-SWCNTs, and Pl-PEG 5000 SWCNTs. An intense peak was observed at $\sim 1595 \mathrm{~cm}^{-1}$ (G band), which is a tangential vibrational mode, and a weaker peak at $\sim 1344 \mathrm{~cm}^{-1}$ (the mid-energy vibrational band, or D band), which is a disorder-induced band. Both bands are characteristic to all graphitic materials. D band is indicative of both the presence of defective sites on SWCNTs (including functional attachments) and carbonaceous impurities. Similarly, the

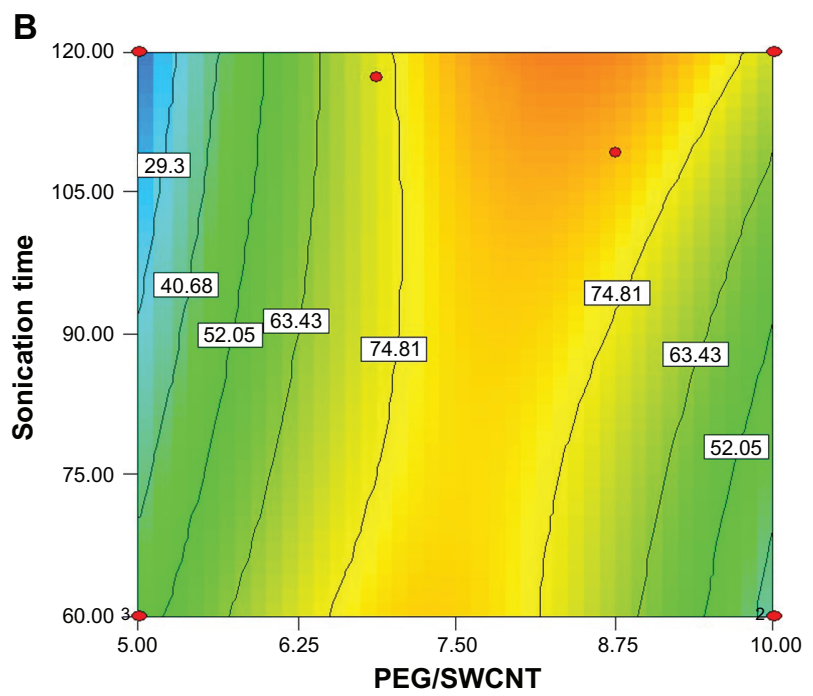

Figure 4 Contour plots of single-walled carbon nanotubes (SWCNTs) functionalized with phospholipid-polyethylene glycol ( $\left.\mathrm{PI}_{-} \mathrm{PEG}\right)$ 2000-NH $\mathrm{f}_{2}$ for $\mathbf{A}$ ) aqueous concentration and B) loading efficiency. 

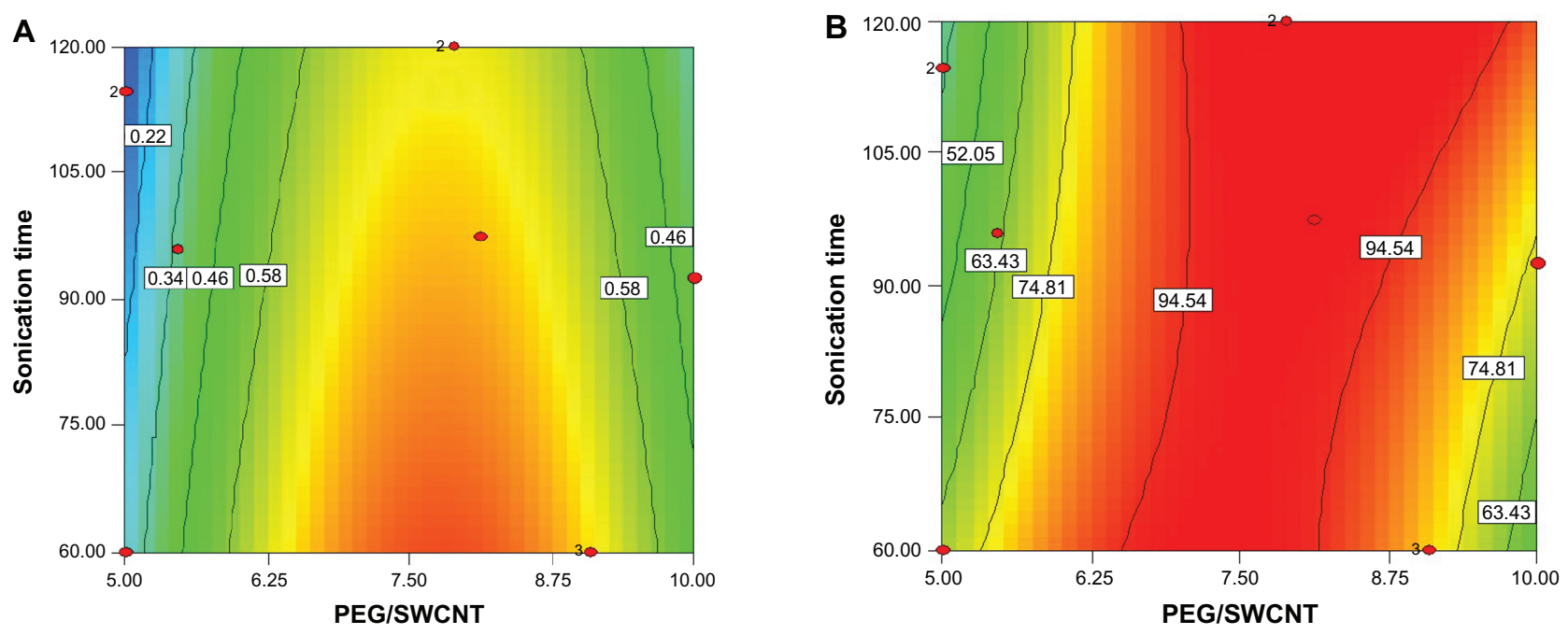

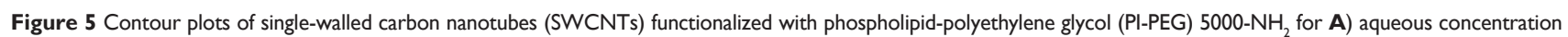
and B) loading efficiency.

presence of functional groups on the walls of SWCNTs is inferred from the $\mathrm{D}$ band. The ratio of intensity of the $\mathrm{G}$ band $\left(\mathrm{I}_{\mathrm{G}}\right)$ to $\mathrm{D}$ band $\left(\mathrm{I}_{\mathrm{D}}\right)$ is often used as an estimate of carbonaceous impurity content and defect site density. The second-order $\mathrm{G}^{\prime}$ band at $2676 \mathrm{~cm}^{-1}$ and a broad peak below $300 \mathrm{~cm}^{-1}$ result from the radial movement of carbon atoms, which are characteristic Raman signals of SWCNTs. Raman spectroscopy is an effective technique to monitor the dispersion state of SWCNTs in solution. The dispersion state is shown by a decrease in $I_{G} / I_{D}$ ratio. The ratio of $I_{G} / I_{D}$ for
SWCNTs is 9.09. After functionalization, the $\mathrm{I}_{\mathrm{G}} / \mathrm{I}_{\mathrm{D}}$ ratio of SWCNTs decreased from 9.09 to 8.69 and 6.66 for Pl-PEG 2000-SWCNTs and Pl-PEG 5000-SWCNTs, respectively, indicating the presence of noncovalently bound moieties on the nanotube framework.

Functionalization optimization was performed by setting a combination of independent variables that would provide optimum results for both responses simultaneously. Constraints chosen for the dependent and independent variables are shown in Table 2. It should be noted that both

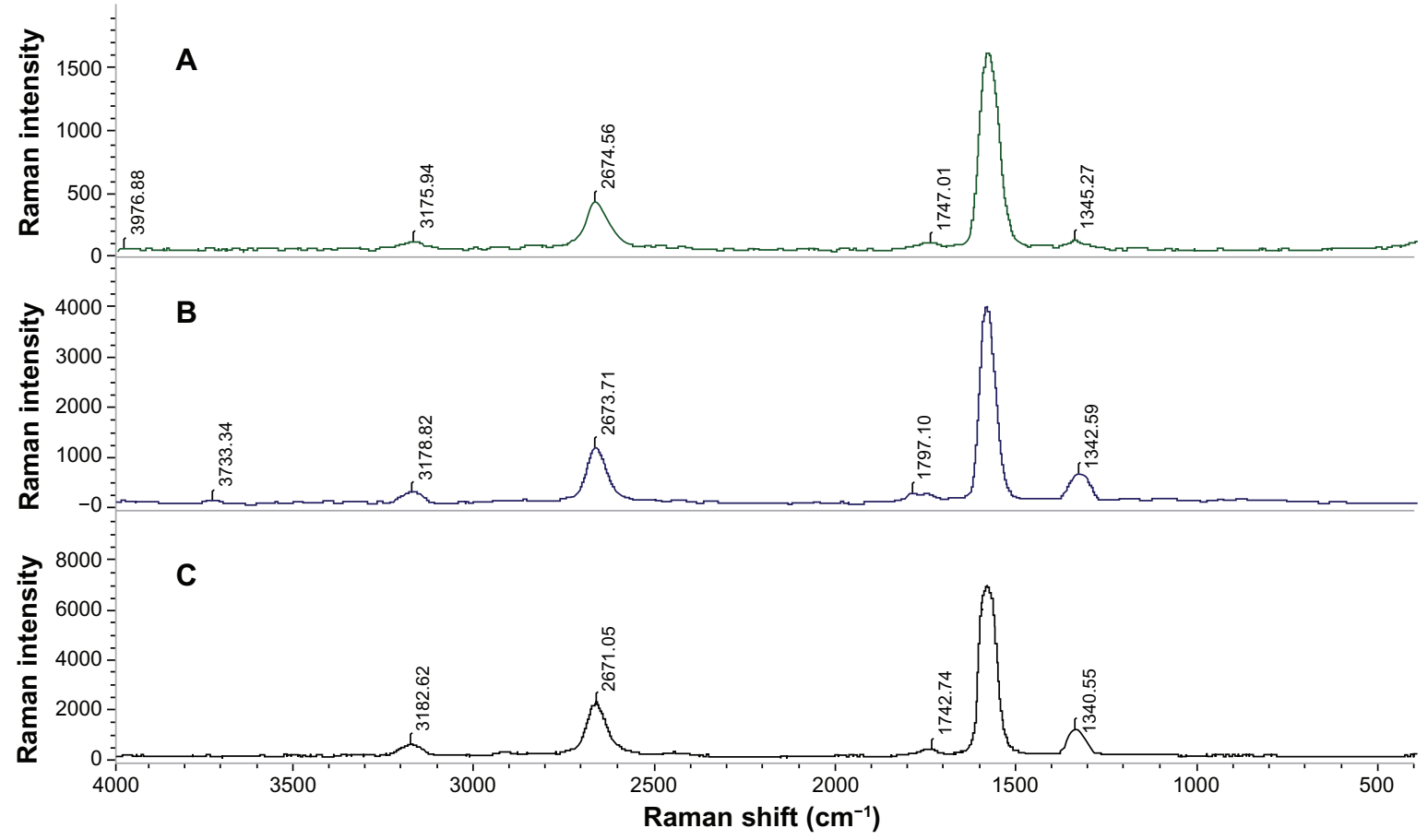

Figure 6 Raman spectra of A) pure single-walled carbon nanotubes (SWCNTs), B) phospholipid-polyethylene glycol (PI-PEG) 5000-SWCNTs, and C) PI-PEG 2000-SWCNTs. 
Table 3 Optimum solutions and the corresponding responses proposed on the basis of optimum responses ${ }^{\mathrm{a}}$

\begin{tabular}{lllll}
\hline Solution & PEG/SWCNT & $\begin{array}{l}\text { Sonication time } \\
\text { (minute) }(\mathbf{B})\end{array}$ & $\begin{array}{l}\text { SWCNT aqueous } \\
\text { concentration }(\mathbf{m g} / \mathbf{m L})\end{array}$ & $\begin{array}{l}\text { Loading } \\
\text { efficiency }(\%)\end{array}$ \\
\hline 1 & 7.79 & 60 & 0.84 & 98.11 \\
2 & 7.86 & 60 & 0.84 & 97.35 \\
3 & 7.68 & 60 & 0.84 & 97.32 \\
4 & 7.34 & 60 & 0.84 & 97.54 \\
\hline
\end{tabular}

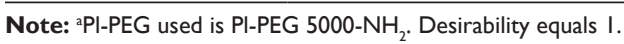

Abbreviations: PI-PEG, phospholipid-polyethylene glycol; SWCNT, single-walled carbon nanotube.

responses were set to be maximized, and the desirability of optimized solutions was kept constant at 1 . Optimum solutions proposed are listed in Table 3, and the related contour plot is depicted in Figure 7. With the conditions set for this process, only samples containing Pl-PEG 5000 were proposed as the optimum solutions.

By reviewing the proposed solutions and considering the related contour plot (Figure 7), it could be concluded that the aqueous solubility of $0.84 \mathrm{mg} / \mathrm{mL}$ and loading efficiency of nearly 98\% were achievable at Pl-PEG 5000/SWCNT weight ratios of 7.3-7.8 and sonication times of 60 minutes.

To obtain a quantitative picture of the mass content of Pl-PEGs in $f$-SWCNTs, TGA was performed for both functionalized and nonfunctionalized SWCNTs. As depicted in Figure 8 , TGA results indicate a gradual distinct mass loss of around $52.42 \%$ at temperatures between $200^{\circ} \mathrm{C}$ and $500^{\circ} \mathrm{C}$. Comparison between the two samples indicates that only $4.7 \%$ mass loss is due to SWCNT impurities, and the rest (ie, $47.72 \%$ ) is due to the presence of Pl-PEGs. Determination of Pl-PEG loading on SWCNTs makes it possible to estimate the fraction of carbon atoms that are functionalized.

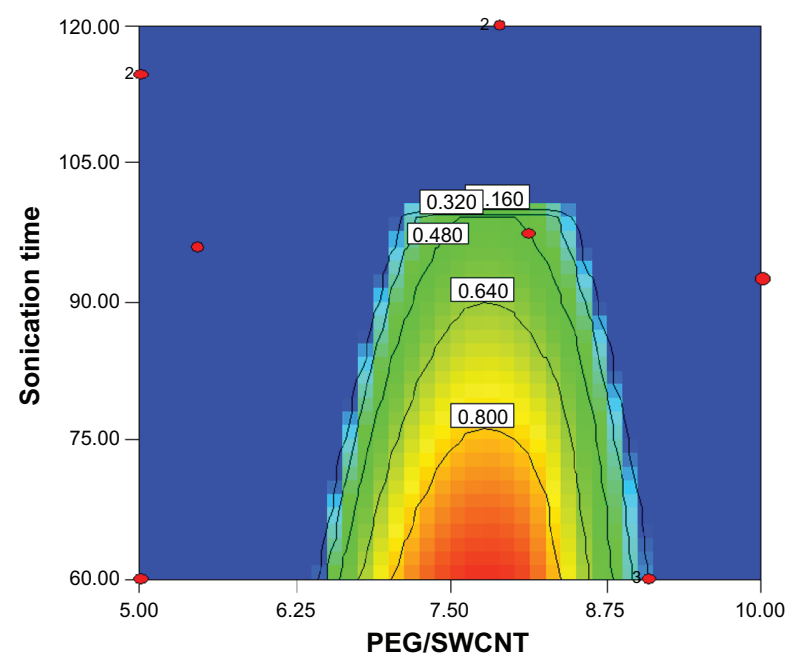

Figure 7 Contour plot of optimized samples constructed with phospholipidpolyethylene glycol (PI-PEG) 5000- $\mathrm{NH}_{2}$, displaying optimum results for both responses, simultaneously.

Abbreviation: SWCNT, single-walled carbon nanotube.
Considering the molecular weight of Pl-PEG 5000 (5679), the degree of functionality can be calculated as a molar functionality using the following equation. Results showed that $0.23 \%$ of the carbons in the SWCNTs are functionalized with Pl-PEGs.

Molar functionality $=$

$$
\frac{(\text { total mass loss } \times 12)}{\text { Mw of SWCNT } \times \text { mass loss related to Pl-PEGs }} \times 100
$$

Finally, in order to confirm the desirability of the optimized samples and, in turn, validate the optimization process, four proposed samples were experimentally prepared and the two responses were evaluated (Table 4). Verification of optimization was confirmed by calculating the Euclidean distance (Ed) using the following equation: ${ }^{15}$

$$
\mathrm{Ed}=\left[\sum_{i}\left(\mathrm{P}_{i}-\mathrm{O}_{i}\right)^{2}\right]^{1 / 2}
$$

where $\mathrm{P}_{i}$ and $\mathrm{O}_{i}$ are predicted and observed values on response $i$, respectively, and the summation is the overall responses. As seen from Table 4, low Ed demonstrated the closeness between the predicted and observed responses.

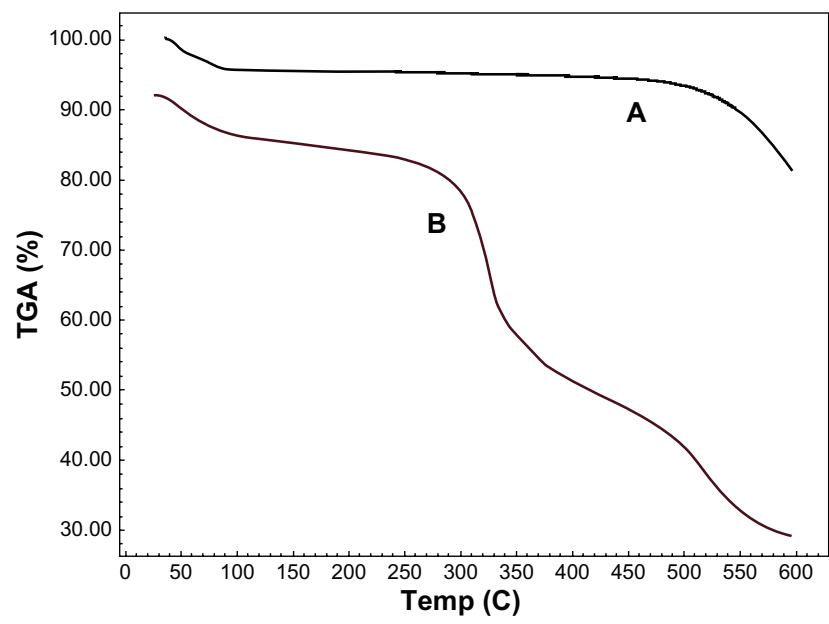

Figure 8 Thermogravimetric analysis (TGA) graphs of A) nonfunctionalized pure single-walled carbon nanotubes (SWCNTs) and B) phospholipid-polyethylene glycol 5000-SWCNTs. 
Table 4 Validation of optimization process

\begin{tabular}{|c|c|c|c|c|c|c|c|c|}
\hline \multirow[t]{2}{*}{ Sample } & \multirow[t]{2}{*}{$\begin{array}{l}\text { PEG/SWCNT } \\
\text { weight ratio }\end{array}$} & \multirow[t]{2}{*}{$\begin{array}{l}\text { Sonication time } \\
\text { (minute) }\end{array}$} & \multirow[t]{2}{*}{$\begin{array}{l}\text { PI-PEG } \\
\text { type }\end{array}$} & \multicolumn{2}{|c|}{$\begin{array}{l}\text { SWCNT aqueous } \\
\text { concentration } \\
(\mathrm{mg} / \mathrm{mL})\end{array}$} & \multicolumn{2}{|c|}{$\begin{array}{l}\text { Loading } \\
\text { efficiency (\%) }\end{array}$} & \multirow[t]{2}{*}{$\mathbf{E d}^{\mathbf{a}}$} \\
\hline & & & & $\mathbf{P}_{i}^{\mathrm{b}}$ & $\mathbf{O}_{i}^{c}$ & $\mathbf{P}_{i}$ & $O_{i}$ & \\
\hline 1 & 7.79 & 60 & PI-PEG 5000 & 0.84 & 0.81 & 98.11 & 92.70 & 5.41 \\
\hline 2 & 7.86 & 60 & PI-PEG 5000 & 0.84 & 0.78 & 97.35 & 96.00 & 1.35 \\
\hline 3 & 7.68 & 60 & PI-PEG 5000 & 0.84 & 0.75 & 97.32 & 93.00 & 4.32 \\
\hline 4 & 7.34 & 60 & PI-PEG 5000 & 0.84 & 0.82 & 97.54 & 94.50 & 3.04 \\
\hline
\end{tabular}

Notes: aEuclidean distance; 'P Predicted value; 'Observed value.

Abbreviations: PI-PEG, phospholipid-polyethylene glycol; SWCNT, single-walled carbon nanotube.

SEM images of pure SWCNTs and a sample of Pl-PEG 5000-SWCNTs (sample 2, Table 4) confirm the attachment of shortened SWCNTs with Pl-PEGs in comparison with pure SWCNTs, which appear as interconnected networks of tubes (Figure 9).

Dispersibility of CNTs can vary from insoluble (sedimented) to soluble (or dispersed). ${ }^{8} f$-SWCNTs prepared in this investigation can be assigned to the soluble category, as a stable black/brown uniform color, characteristic of a homogeneous dispersion, with no signs of phase separation was observed for 2 months after dispersion by sonication
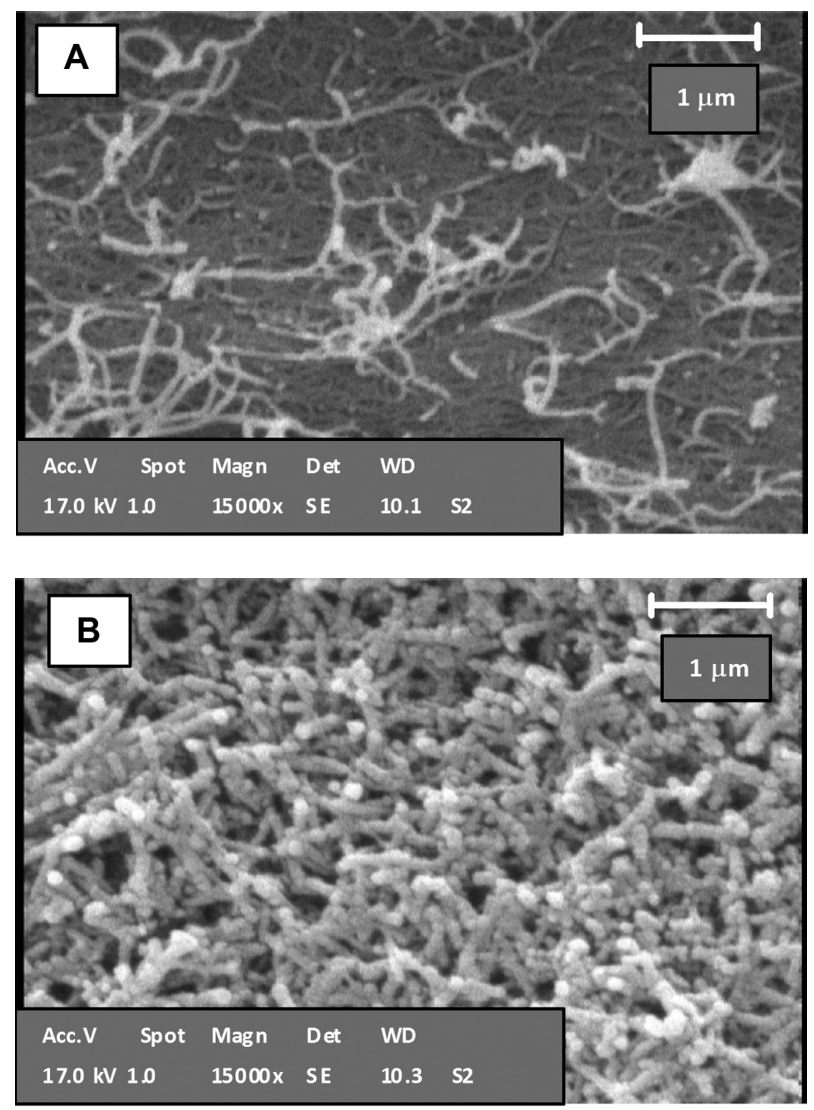

Figure 9 Scanning electron microscopy images of A) nonfunctionalized pure single-walled carbon nanotubes (SWCNTs) and B) phospholipid-polyethylene glycol 5000-SWCNTs.
(Figure 10). It is suggested that the PEG chains were functionalized with the terminal amine (Pl-PEG-NH $\left.{ }_{2}\right)$ group, resulting in positive interaction with water. As stated previously, the solubility (a measure of individually dispersed SWCNTs) was measured by UV spectrophotometry.

\section{Discussion}

CNTs, either single walled or multiple walled, are an important new class of technological materials that have novel applications. ${ }^{16}$ Despite various physical properties and remarkable features, surface characteristics of CNTs make them chemically inert and incompatible with nearly all organic and inorganic solvents. Chemical characteristics are attributed to the smooth surface with no hanging bonds but rather the hydrophobic character of the sidewalls, coupling the individual tubes, and the formation of bundles or aggregates. ${ }^{7}$

CNTs have been reported to offer a promising strategy for improving body distribution, prolonging blood circulation,

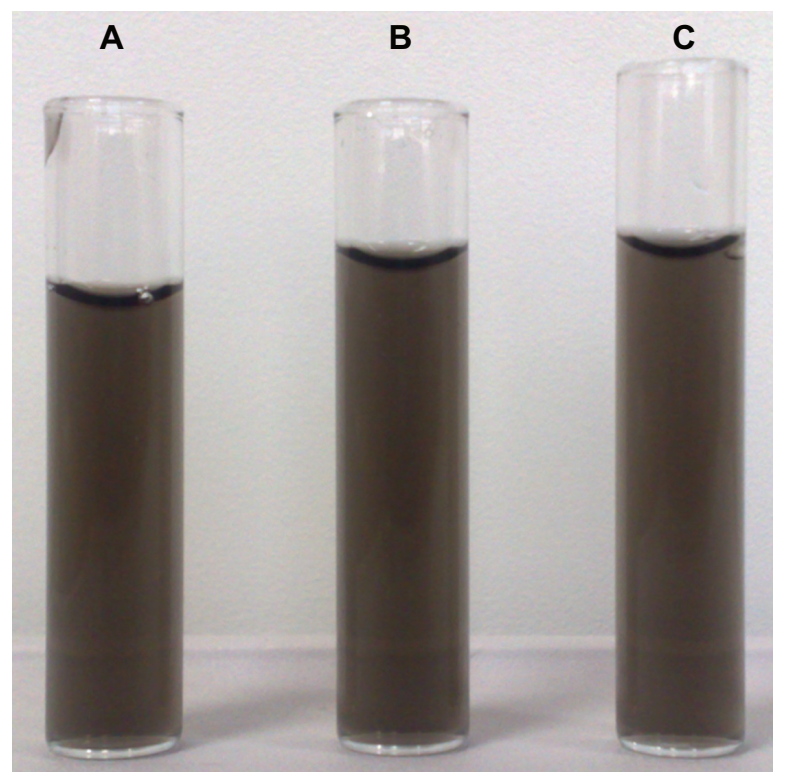

Figure 10 Homogenous dispersion of functionalized single-walled carbon nanotubes (sample from Table I, run 14) A) at the time of preparation, B) after storage for I month, and C) after storage for 2 months. 
and internalizing various drugs in cells, provided that they are well functionalized. ${ }^{17} \mathrm{CNT}$ s possess extremely low solubility. Therefore, pure CNTs are not capable of circulating appropriately in biological fluids. Surface functionalization through chemical reactions or attachment of solubilizing side chains either covalently or noncovalently makes CNTs more dispersible or biocompatible with physiological systems and hence reduces their toxicity compared with pristine CNTs. On the other hand, once solubilized, CNTs efficiently cross cell membranes, and, as a result, various molecules attached to the surface of the SWCNT can be internalized., ${ }^{4,17-22}$ Following this process, the weak attraction forces (van der Waals forces) between the individual tubes are eliminated, leading to dispersion being achieved. .,20,23 $^{2}$

The most common covalent reaction is the chemical modification of the graphene surface by oxidation, followed by organic modification with hydrophilic substances. ${ }^{24-26}$ With this strategy, the defective carbon atoms at the end of CNTs or on the sidewall can be oxidized to generate carboxylic acid groups or carboxylated fractions, enabling various molecules to be grafted to the surface of CNTs. The functionalization of CNTs can also be achieved by noncovalent surface coating by low-molecular-weight surfactants, surface wrapping with polymers, or through $\pi-\pi$ stacking interaction between the aromatic rings of the loaded materials and $\pi$-electrons of graphite layer on the surface of CNTs. ${ }^{24,27,28}$

In this research, noncovalent functionalization of SWCNTs by Pl-PEG 2000 and Pl-PEG 5000 was developed to meet the aforementioned requirements. PEG is increasingly used for various purposes in biomedical and pharmaceutical applications, due to its biocompatibility and appropriate aqueous solubility. Pl-PEG conjugates are amphiphilic compounds with hydrophobic chains and hydrophilic head groups. The two hydrocarbon chains can strongly attach to the nanotube surface with the hydrophilic PEG chain extending into the aqueous phase, imparting desirable water solubility and biocompatibility. ${ }^{24,29}$ It has been shown that the PEGfunctionalized SWCNTs exhibited excellent stability without agglomeration in biological media. ${ }^{6}$ In addition to improved water solubility, an increase in blood circulating time and a decrease in uptake by reticuloendothelial system have also been reported. ${ }^{6}$ Pl-PEG 2000 was selected because it is expected to have more interactions with hydrophobic structures of cell membranes and therefore higher cell uptake due to the exposure of the hydrophobic surface. However, P1-PEG 5000 was also investigated in this study, as it is expected to reduce the nonspecific binding of serum proteins, compared with P1-PEG 2000. It should be noted that P1-PEGs with one available amine group were studied to reduce the possible steric hindrance, compared with multiarmed Pl-PEGs, for drug conjugation in the next step of this research. The results of this work showed that our noncovalent functionalization protocol could considerably increase aqueous solubility, which is an essential criterion in the design of a CNT-based drug delivery system. Our future studies will be focused on the use of free amine functional groups positioned at the hydrophilic PEG terminal to conjugate drug molecules.

\section{Conclusion}

SWCNTs have low solubility and are difficult to manipulate in aqueous media. Functionalization approaches have opened new horizons for solubilizing and dispersing CNTs in water and therefore reduce their toxicity. We planned this study to PEGylate SWCNTs with PEG through hydrophobic interaction between CNT sidewalls and the hydrocarbon chain of the phospholipid component of a Pl-PEG moiety in order to obtain noncovalent functionalized P1-PEG-SWCNTs. The good agreement between the predicted and observed responses indicates the adequacy and applicability of experimental design methods for solubility optimization. These nanostructures might be used to graft various drugs through the PEG chains and therefore could be considered as promising nanocarriers for drug delivery.

\section{Acknowledgment}

The authors would like to thank Iran National Science Foundation (INSF) and Research Deputy of Shaheed Beheshti University of Medical Sciences in Iran for the financial support for this research. This study is a part of $\mathrm{PhD}$ thesis of Naghmeh Hadidi, proposed and approved by the School of Pharmacy, Shaheed Beheshti University of Medical Sciences, Iran.

\section{Disclosures}

The authors report no conflicts of interest in this work.

\section{References}

1. Bekyarova E, Ni Y, Malarkey EB, et al. Applications of carbon nanotubes in biotechnology and biomedicine. J Biomed Nanotechnol. $2005 ; 1: 3-17$

2. Iijima S. Helical microtubes of graphitic carbon. Nature. 1991;354:56-58.

3. Liang F, Chen B. A review on biomedical applications of single-walled carbon nanotubes. Curr Med Chem. 2010;17:10-24.

4. Foldvari M, Bagonluri M. Carbon nanotubes as functional excipients for nanomedicines: II. Drug delivery and biocompatibility issues. Nanomed. 2008;4:183-200.

5. Khazaei A, Soltani Rad MN, Kiani Borazjani M. Organic functionalization of single-walled carbon nanotubes (SWCNTs) with some chemotherapeutic agents as a potential method for drug delivery. Int J Nanomed. 2010;5:639-645. 
6. Zhuang L, Chen K, Davis C, et al. Drug delivery with carbon nanotubes for in vitro cancer treatment. Cancer Res. 2008;16:6652-6660.

7. Zhang Y, Yuhong B, Yan B. Functionalized carbon nanotubes for potential medicinal applications. Drug Discov Today. 2010;15:428-435.

8. Foldvari M, Bagonluri M. Carbon nanotubes as functional excipients for nanomedicines: I. Pharmaceutical properties. Nanomed. 2008;4: $173-182$.

9. Tran PA, Zhang L, Webster TJ. Carbon nanofibers and carbon nanotubes in regenerative medicine. Adv Drug Deliv Rev. 2009;61:1097-1114.

10. Mehra NK, Jain AK, Lodhi N, et al. Challenges in the use of carbon nanotubes for biomedical applications. Crit Rev Ther Drug Carrier Sys. 2008;25:169-206.

11. Jain AK, Dubey V, Mehra NK, et al. Carbohydrate-conjugated multiwalled carbon nanotubes: development and characterization. Nanomed. 2009;5:432-442.

12. Liu Z, Tabakman S, Chen Z, et al. Preparation of carbon nanotube bioconjugates for biomedical applications. Nature Protocol. 2009;4: $1372-1382$.

13. Zhao B, Hu H, Yu A, et al. Synthesis and characterization of water soluble single-walled carbon nanotube graft copolymers. $J \mathrm{Am}$ Chem Soc. 2005; 127:8197-8203.

14. Habeeb AFSA. Determination of free amino groups in proteins by trinitrobenzene sulfonic acid. Anal Biochem. 1996;14:328-336.

15. Lim CP, Quek SS, Peh KK. Prediction of drug release profiles using an intelligent learning system: an experimental study in transdermal iontophoresis. J Pharm Biomed Anal. 2003;31:159-168.

16. Kishore AS, Surekha P. Murthy PB. Assessment of the dermal and ocular irritation potential of multi-walled carbon nanotubes by using in vitro and in vivo methods. Toxicol Lett. 2009;191:268-274.

17. Dhar S, Liu Z, Thomale J, et al. Targeted single wall carbon nanotube mediated $\mathrm{Pt}(\mathrm{IV})$ prodrug delivery using folate as a homing device. $\mathrm{JAm}$ Chem Soc. 2008;130:11467-11476.
18. Sayes CM, Liang F, Hudson JL, et al. Functionalization density dependence of single-walled carbon nanotubes cytotoxicity in vitro. Toxicol Lett. 2006;161:135-142.

19. Zhang LW, Zeng L, Barron AR, Monteiro-Riviere NA. Biological interactions of functionalized single-wall carbon nanotubes in human epidermal keratinocytes. Int J Toxicol. 2007;26:103-113.

20. Dyke CA, Tour JM. Overcoming the insolubility of carbon nanotubes through high degrees of sidewall functionalization. Chem Eur J. 2004;1: $1117-1124$.

21. Fernando KA, Lin Y, Sun YP. High aqueous solubility of functionalized single-walled carbon nanotubes. Langmuir. 2004;10:812-817.

22. Peng H, Alemany LB, Margrave JL, et al. Side wall carboxylic acid functionalization of single-walled carbon nanotubes. $\mathrm{J} \mathrm{Am} \mathrm{Chem} \mathrm{Soc.}$ 2003;125:15174-15182.

23. Lacerda L, Bianco A, Prato M. Carbon nanotubes as nanomedicines: from toxicology to pharmacology. Adv Drug Deliv Rev. 2006;58: $1460-1470$.

24. Liu Z, Tabakman S, Welsher K, et al. Carbon nanotubes in biology and medicine: in vitro and in vivo detection, imaging and drug delivery. Nano Res. 2009;2:85-120.

25. Niyogi S, Hamon MA, Hu H, et al. Chemistry of single-walled carbon nanotubes. Acc Chem Res. 2002;35:1105-1113.

26. Rosca ID, Watari F, Uo U, et al. Oxidation of multi-walled carbon nanotubes by nitric acid. Carbon. 2005;43:3124-3131.

27. Richard C, Balavoine F, Schultz P, et al. Supramolecular self-assembly of lipid derivatives on carbon nanotubes. Science. 2003;300:775-778.

28. Wang H, Zhou W, Ho DL, et al. Dispersing single-walled carbon nanotubes with surfactants: a small angle neutron scattering study. Nano Lett. 2004;4:1789-1793.

29. Liu Z, Sun X, Nakayama N, et al. Supramolecular chemistry on watersoluble carbon nanotubes for drug loading and delivery. ACS Nano 2007;1:50-56.
International Journal of Nanomedicine

\section{Publish your work in this journal}

The International Journal of Nanomedicine is an international, peerreviewed journal focusing on the application of nanotechnology in diagnostics, therapeutics, and drug delivery systems throughout the biomedical field. This journal is indexed on PubMed Central, MedLine, CAS, SciSearch ${ }^{\circledR}$, Current Contents ${ }^{\circledR} /$ Clinical Medicine,

\section{Dovepress}

Journal Citation Reports/Science Edition, EMBase, Scopus and the Elsevier Bibliographic databases. The manuscript management system is completely online and includes a very quick and fair peer-review system, which is all easy to use. Visit http://www.dovepress.com/ testimonials.php to read real quotes from published authors. 